\title{
Stress among emergency medicine residents during the COVID-19 pandemic: A qualitative study
}

\section{Dear Editor,}

The COVID-19 pandemic has disrupted medical education $^{1,2}$ and distressed clinicians. ${ }^{3,4}$ Understanding the impact of this pandemic on emergency medicine (EM) residents' experience of stress will allow for more effective interventions to aid residents, while reducing attrition and its impact on pandemic response.

We present our qualitative study, guided by a theoretical framework underpinned by empirical evidence, which explicitly explored residents' experiences of stress during the pandemic, important and modifiable underlying factors, and how this can guide prioritisation of limited resources to aid residents. Ethics approval was obtained (DSRB reference number 2020/00523).

We framed our study using the Lazarus and Folkman transactional model of stress, ${ }^{5}$ given its strong empirical foundations. In this model, stress is a relationship between the individual and the environment that is cognitively appraised as exceeding the individual's resources and threatening well-being.

Cognitive appraisal comprises 2 parts. Primary appraisal determines what is at stake, i.e. whether it is irrelevant, beneficial or threatening to the individual. Commitments influence primary appraisal, with deeper commitments increasing potential threat and driving coping efforts. Secondary appraisal evaluates coping options based on availability, and ability to accomplish the intended outcome and apply them effectively. Coping is the process of managing the demands of the personenvironment relationship and the emotions generated.

The study was conducted according to the COnsolidated criteria for REporting Qualitative research (COREQ) checklist $^{6}$ and a phenomenological paradigm. Purposive sampling was employed to ensure representation across sites and levels of training. Sampling was deemed complete when thematic saturation was achieved, i.e. no new themes emerged. There were 92 EM residents in Singapore during the study period (October-November 2020); all were eligible and invited for recruitment via email. Twenty-six residents were recruited, and 4 focus group discussions (FGDs) were conducted. FGDs lasted 49-65 minutes. No residents dropped out after recruitment. Written informed consent was obtained from all participants. Participation was voluntary and none received compensation.
FGDs were conducted by 2 study team members and in groups of 5-8 participants, through video calls at home. One study team member was the primary moderator, and the other was an observer and notetaker. To foster psychological safety, the residents were grouped according to level of training, with FGD moderators from a different resident training site. FGDs were semi-structured with an interview guide derived from consensus among the study team, guided by literature review. Questions explored participants' experiences of stress in general during the pandemic, cognitive appraisals and coping strategies. Audio of the interviews were recorded and transcribed by the primary moderator.

Each transcript was first analysed using open coding by study team members who worked in pairs. As themes emerged from analysis of the first 2 transcripts, a coding template that included major themes was produced based on consensus among the study team, and applied to all transcripts. Further discussion refined themes to encompass the data as fully as possible. Transcripts and codes were returned for member checking by the participants.

Three major themes emerged and are described with illustrative quotes in Table 1.

Theme 1: Stress appraisal was influenced by adequacy of resources that residents valued. Such resources included information about COVID-19, continued exposure to non-pandemic cases for learning, manpower commensurate with workload, isolation facilities and workflows, personal protective equipment and job security. When these resources were plentiful, gratitude was induced. The pandemic was a unique learning opportunity, but with limited value that eroded over time.

Theme 2: Commitments influence cognitive appraisal. The pandemic was an opportunity to fulfill residents' commitment to professional identity. This was reinforced by senior staff role modelling commitment to colleagues. Commitments to next of kin contributed to stress and feelings of isolation for residents who had migrated for work and could not return due to lockdowns.

Theme 3: Effectiveness of individual and system coping strategies. Online learning was appraised as convenient, though sometimes ineffective due to difficulty in maintaining attention, and inability to 
Table 1. Themes, sub-themes and quotes illustrating the residents' experiences

\begin{tabular}{lll}
\hline \multicolumn{1}{c}{ Theme } & \multicolumn{1}{c}{ Sub-theme } & \multicolumn{1}{c}{ Quotes } \\
\hline $\begin{array}{l}\text { Stress appraisal is influenced } \\
\text { by the adequacy of resources } \\
\text { valued by residents }\end{array}$ & $\begin{array}{l}\text { Information about } \\
\text { the disease }\end{array}$ & $\begin{array}{l}\text { Participant 2-4: "Stressful to work in seeing COVID-19 cases, because at that period } \\
\text { of time, we don't know how deadly this disease is, how fast it spreads." }\end{array}$ \\
& &
\end{tabular}

Continued exposure to non-pandemic cases for learning
Participant 2-3: “...taken away from our main ED (emergency department) practice and we are not able to see the general population of cases; instead we are seeing foreign workers or the URTIs (upper respiratory tract infections) that are referred from the GPs (general practitioners) and I would feel that it affects our learning because we only know how to clear URTI cases now."

Participant 1-3: "When you are in the whole COVID situation, no one really thinks about training that much anymore. You just deal with the load."

Procedural learning opportunities

Participant 4-4: "Hoping for greater yield of procedures, but...less opportunities for chest drains because less patients coming in with pleural effusions."

Manpower commensurate with workload

Participant 1-3: "When I was at the dirty ICU (intensive care unit), our admission rate was 3 times of the clean team, but at the same time, the ratio of the staffing is the same, one to one. No one asked us, "Are you all overloaded? Are you all ok?"”

Isolation workflows and facilities

Participant 4-6: "In some other institution(s), I know that they have special pathway(s) for them to transfer the patient from the ED to the ICU... in my hospital we don't have this... they will definitely go through the general walkway and might have some contact with the rest of the patient(s)."

Participant 2-4: "What we don't want is getting a call few hours later or the next morning, "you just exposed 6 healthy patients to 1 COVID-19 patient."”

Personal protective equipment (PPE) and job security

Participant 4-1: "I have friends in (another country)... They are telling me that they see COVID suspects just with a surgical mask... The resources we have are really great compared to what my friends and siblings have out there in different countries."

Participant 1-5: "There were so many people out there who either lost their jobs or (who were) stuck at home. They were essentially almost like locked up at home because everybody cannot go out, while I still can go to my job daily."

Pandemic as a learning opportunity

Participant 3-4: "A very wonderful opportunity for the ED people to learn. How many of us see a pandemic once in our lifetime, to actually see it run on the ground?"

Commitments influence cognitive appraisal
Opportunity to fulfill

commitment to professional identity

Inspired by senior staff role modelling commitment to colleagues

Threat arising from commitment to connections with next of kin

Effectiveness of individual and system coping strategies
Deteriorating appraisal of the pandemic over time
Online learning as beneficial

Drawbacks of online learning
Participant 2-5: "In the first 1 or 2 months, everyone is very fired up... so initially we didn't really feel the pain... We started to feel it around the third month, because we lose all this training, then we never get smarter, we just keep seeing COVID patients."

Participant 1-1: "This is our job. We signed up for this. As A\&E (accident and emergency) people, we are frontline for this. I don't think any of us would have chosen otherwise."

Participant 1-3: "If you see certain seniors, willing to be busy and getting down to do the dirty shift, or volunteer to do more of the dirty shifts with you, then you are more inspired... What's most motivating is the feeling that everyone is doing this together."

Participant 4-5: “Because I'm not a local, I've not actually been home for a year.. I have times that I dearly miss my family but unfortunately I can't do much about it."

Participant 4-1: "Definitely very convenient... I like my big screen in my room, having my own coffee... saves me a lot of travelling time."

Participant 3-1: "I personally prefer physical classroom or auditorium teaching. I find it hard to concentrate on online Zoom teaching."

Participant 3-3: "Some things cannot be replaced by the online teaching like the procedural skills and simulation."

Importance of self-regulation: time management
Participant 4-6: "For those people who are not compliant, this will be a bit tough because there is a lack of supervision...there're pros and cons... If the resident can manage themselves quite well, time management is good, I think it's very good for learning." 
Table 1. Themes, sub-themes and quotes illustrating the residents' experiences (Cont'd)

\begin{tabular}{|c|c|c|}
\hline Theme & Sub-theme & Quotes \\
\hline & $\begin{array}{l}\text { Importance of self-regulation: } \\
\text { strategic planning and } \\
\text { independently seeking out } \\
\text { teachers who had adapted } \\
\text { well to the new educational } \\
\text { landscape }\end{array}$ & $\begin{array}{l}\text { Participant 1-4: "Those who did very well, they approached us about how to go } \\
\text { about doing the tutorials, how to improve the tutorials... I just prioritised the } \\
\text { tutors I felt were optimising the Zoom tutorial sessions and contributing the } \\
\text { most to help me." }\end{array}$ \\
\hline & $\begin{array}{l}\text { Communication between } \\
\text { faculty and residents } \\
\text { aided coping }\end{array}$ & $\begin{array}{l}\text { Participant } 3-5: \text { "... received a call from my PD (programme director)...they } \\
\text { would try their best to help and we can talk to them anytime we } \\
\text { have a problem." }\end{array}$ \\
\hline & $\begin{array}{l}\text { Drawing strength from } \\
\text { connections with family } \\
\text { and friends }\end{array}$ & $\begin{array}{l}\text { Participant 1-1: "It's really helpful to have a good work family or friends around } \\
\text { so that was a supporting factor. Especially family members." }\end{array}$ \\
\hline & $\begin{array}{l}\text { Value of safe social } \\
\text { interaction with colleagues }\end{array}$ & $\begin{array}{l}\text { Participant 2-3: "I actually enjoyed and looked forward to going to work because } \\
\text { that was the most social interaction I probably had physically, and it was only } \\
\text { possible because we were all in PPE and we don't need to stay like } 5 \mathrm{~m} \text { away } \\
\text { from each other and then we can actually sit around the seniors' counter and talk } \\
\text { like normal." (The rest of the group laughs.) }\end{array}$ \\
\hline & & $\begin{array}{l}\text { Participant 4-6: "Our residents will still do exercise together...in our meeting, } \\
\text { but with social distancing. So, we HIIT (high intensity interval training) together, } \\
\text { do cardio together (laughs)." }\end{array}$ \\
\hline
\end{tabular}

replace simulation or procedural skills training. Residents recognised the need for self-regulation skills, such as strategic planning and time management to cope with the new educational landscape, characterised by greater learner independence. Communication with faculty members and loved ones, and social interaction at work, mitigated feelings of isolation.

Our findings suggest the following targets for intervention to address stress among residents:

1. Optimising use of limited resources by prioritising the most impactful, i.e. those valued by residents. There is potential to transform stress into gratitude, which demonstrates a wider extent of impact that resource adequacy has compared to previous studies. ${ }^{3,4}$

2. A deliberately planned balance between pandemic and non-pandemic case exposure, to extract learning value from the pandemic, while maintaining sufficient exposure to business-as-usual cases for core clinical training.

3. Role modelling by senior clinicians, to strengthen commitment to professional identity and colleagues as a protective factor.

4. Deliberately fostering social interactions in a physically safe manner between residents, colleagues and loved ones.
5. Improving the effectiveness of online learning through thoughtful implementation based on established principles, ${ }^{7}$ since the future of medical education has likely been permanently altered, ${ }^{8}$ with online learning now increasingly integral. However, as online learning is an inadequate modality in some contexts (e.g. for procedural skills), new ways to safely conduct face-to-face and workplace-based training should be explored.

6. Fostering self-regulation skills, ${ }^{9}$ including but not limited to those highlighted by the participants in our study.

As we were limited to a single country's EM residency programmes, our findings may not be generalisable to other settings with a different disease burden. Interestingly, stigmatisation of healthcare workers was not a prominent theme in this study, in contrast to literature. ${ }^{10}$ Further studies may examine the reasons for this, and whether the factors identified in our cohort are generalisable across different stressful situations, and even across specialties. Measurable effectiveness of the interventions that targeted stressors would also help to ascertain our presented strategies as proven stress management measures for residents.

In conclusion, leadership in residency programmes can focus on targets identified in our study to mitigate stress among residents during a pandemic. 


\section{REFERENCES}

1. Liang ZC, Ooi SBS, Wang W. Pandemics and Their Impact on Medical Training: Lessons From Singapore. Acad Med 2020; 95:1359-61.

2. Lam JSL, Wong TTC, $\mathrm{Ku} \mathrm{CW}$, et al. Impact of COVID-19 on paediatric and OBGYN residency training in Singapore. Ann Acad Med Singap 2021;50:717-20.

3. Shanafelt T, Ripp J, Trockel M. Understanding and Addressing Sources of Anxiety Among Health Care Professionals During the COVID-19 Pandemic. JAMA 2020;323:2133-4.

4. Chor WPD, Ng WM, Cheng L, et al. Burnout amongst emergency healthcare workers during the COVID-19 pandemic: A multi-center study. Am J Emerg Med 2021;46:700-2.

5. Lazarus RS, Folkman S. Stress, appraisal, and coping. New York: Springer; 1984.

6. Tong A, Sainsbury P, Craig J. Consolidated criteria for reporting qualitative research (COREQ): a 32-item checklist for interviews and focus groups. Int J Qual Health Care 2007;19:349-57.

7. Ellaway R, Masters K. AMEE Guide 32: e-Learning in medical education Part 1: Learning, teaching and assessment. Med Teach 2008;30:455-73

8. Goh P, Sandars J. A vision of the use of technology in medical education after the COVID-19 pandemic. MedEdPublish 2020;9:49.
9. Sandars J, Cleary TJ. Self-regulation theory: applications to medical education: AMEE Guide No. 58. Med Teach 2011;33:875-86.

10. Bagcchi S. Stigma during the COVID-19 pandemic. Lancet Infect Dis 2020;20:782.

Matthew Jian Wen Low ${ }^{1}$ MBBS (Hons), Chaoyan Dong ${ }^{2}$ PhD, Daniel Yong Jing Quek ${ }^{3}$ MMed, Jian Hui $\mathrm{Ng}^{3}$ MB Bch, Han Nee Gan ${ }^{4}$ MRCSEd, Thian Phey Lau ${ }^{1}{ }_{\text {MCEM }}$, Jen Heng $\underline{\text { Pek }}{ }^{5}$ MCEM

${ }^{1}$ Emergency Medicine Department, National University Hospital, Singapore

${ }^{2}$ Education Office, Sengkang General Hospital, Singapore

${ }^{3}$ Emergency Department, Tan Tock Seng Hospital, Singapore

${ }^{4}$ Accident and Emergency Department, Changi General Hospital, Singapore

${ }^{5}$ Department of Emergency Medicine, Sengkang General Hospital, Singapore

Correspondence: Dr Matthew Jian Wen Low, Emergency Medicine Department, National University Hospital, Level 4, National University Centre for Oral Health, 9 Lower Kent Ridge Road, Singapore 119085. Email: matthew_low@nuhs.edu.sg 